\title{
Temperature Homogeneity under Selective and Localized Microwave Heating in Structured Flow Reactors
}

\author{
Abhinav Malhotra, ${ }^{\bigcirc}$ Weiqi Chen, ${ }^{\bigcirc}$ Himanshu Goyal, Pedro J. Plaza-Gonzalez, Ignacio Julian, \\ Jose M. Catala-Civera, and Dionisios G. Vlachos*
}

Cite This: https://dx.doi.org/10.1021/acs.iecr.0c05580

Read Online

ACCESS |

山ll Metrics \& More

回 Article Recommendations

Supporting Information

ABSTRACT: Selective heating of different phases of multiphase systems via microwaves can result in energy savings and suppression of side reactions. However, materials properties and operating conditions that maximize temperature gradients are poorly understood. Here we utilize computational fluid dynamics (CFD) computations and temperature measurements in structured flow reactors (monoliths) in a monomodal microwave cavity to assess the temperature difference between the walls and the fluid and develop a simple lumped model to estimate when temperature gradients exist. We also explore the material's thermal and electrical properties of structured reactors for isothermal catalyst conditions. We propose that CFD simulations can be used as a nonintrusive, predictive tool of temperature homogeneity. Importantly, we demonstrate that localized heating in the bed under several conditions rather than selective heating is responsible for the selectivity enhancement. Our results indicate that structured beds made of high thermal conductivity materials avoid arcing and enable temperature homogeneity and low electrical conductivity materials allow microwaves to penetrate the domain.

\section{INTRODUCTION}

Greener chemical manufacturing technologies can combat climate change. ${ }^{1}$ Electromagnetic energy generated from renewable sources is one such technology that can simultaneously enable process intensification and sustainable electrified chemical manufacturing. ${ }^{2-12}$ Electromagnetic radiation, including microwaves, can be deposited volumetrically ${ }^{3,13-18}$ and overcome heat transfer limitations occurring when the reactor is heated by an external energy source. ${ }^{19-21}$ Importantly, microwave heating is also selective due to difference in permittivity of materials. For example, microwave absorbing solids, such as silicon carbide ( $\mathrm{SiC}$ ), absorb and dissipate the electromagnetic energy much more efficiently than "transparent" fluids, such as air. Consequently, solid catalysts' preferential heating can lead to energy savings and improve chemical reaction selectivity. Reported examples of enhanced selectivity include the catalytic alkane dehydrogenation to olefins, ${ }^{10}$ ethylene epoxidation, ${ }^{22}$ and furfural production from sugars. ${ }^{23}$ The extent and the conditions causing temperature differences, especially under flow conditions, remain unclear.

Preferential microwave heating is not always beneficial. It can create hot-spots and temperature inhomogeneity, especially in fixed beds, due to point contacts among particles that concentrate the electromagnetic field. ${ }^{9,24}$ Hot-spots can degrade catalyst performance, e.g., by sintering. ${ }^{25,26}$ Structured reactors are emerging as the choice for eliminating these hotspots. The temperature gradients and hot-spots have also been postulated to underlie 'nonthermal' microwave effects, i.e., effects where electromagnetic-molecule interaction underlies enhanced performance over conventional heating. However, as has been noted previously, ${ }^{27,28}$ without a detailed knowledge of the temperature field, the mechanism leading to the improved performance under microwave heating cannot be inferred.

Accurate measurement of the temperature field under microwave heating is a challenging task. ${ }^{29}$ Thermocouplebased measurements in the microwave-incident region are impossible due to the interaction between probe metals and the electromagnetic field. One can carefully shield and position a thermocouple near but outside the electromagnetic field. For instance, the fluid temperature was measured in situ but outside the electromagnetic field ${ }^{10}$ and postmicrowave power shutdown by inserting the thermocouple into the structured reactor channels. ${ }^{30}$ Fiber optics can mitigate the interaction with the electromagnetic field, but they are currently suitable for temperatures $<300{ }^{\circ} \mathrm{C}$ owing to material limitations. The challenges with point-contact techniques, i.e., fiber optics and thermocouples, can be mitigated using contactless measurement methods, including infrared (IR) sensors and thermal

Special Issue: Enrico Tronconi Festschrift

Received: November 17, 2020

Revised: January 24, 2021

Accepted: January 26, 2021 


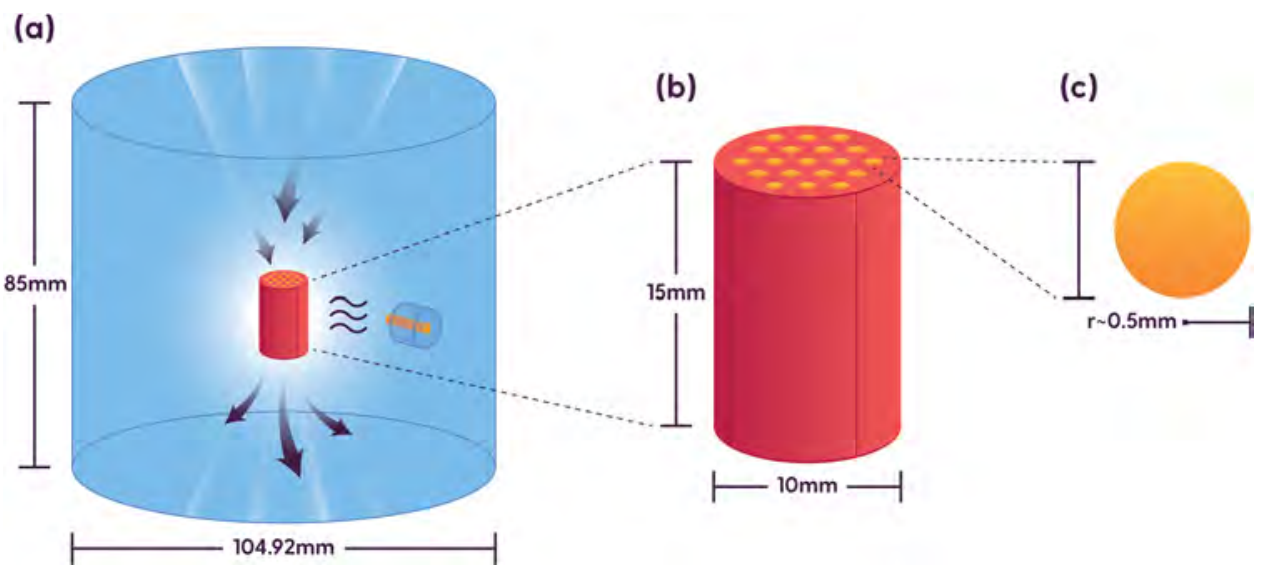

Figure 1. Multiple length scales involved in the microwave heating of structured reactors. Here the heating of an example 3D printed structured bed is shown. (a) The microwave cylindrical cavity with the coaxial port is used to supply energy to (b) a structured bed which has (c) sub mm channels where fluid-solid interaction occurs.

cameras. IR sensors apply to a broader range of temperatures than fiber optics but often underestimate the reaction temperature for an inside-out heating pattern. ${ }^{31}$ Surface temperatures of structured reactors have been successfully visualized even within a quartz tube using thermal cameras operating at optimal transparent IR wavelengths. ${ }^{26,30}$ However, the temperature of the fluids within the channels remains currently beyond the experimental grasp. Simultaneous experimental measurement of both solid and fluid temperatures within structured reactors remains a challenge. Predictive models can help overcome this challenge by providing insights into the temperature field inhomogeneity $^{32-34}$ and methods for engineering it.

In this work, we model the thermal transport in structured (monolith) reactors heated with microwave irradiation in a monomodal cylindrical cavity under flow conditions using computational fluid dynamics (CFD) and conduct corresponding experimental measurements to understand the solid-fluid temperature field. The model is first validated using thermal measurements on a solid cylinder and a monolith without flow. Next, the spatial temperature profile is simulated and measured to understand the role of flow and fluid properties in sustaining a temperature difference through the monolith. Finally, an analytical model is introduced to rationalize the results. We also explore the selection of materials of structured reactors for chemical manufacturing. Our work provides insights into microwave-heated structured reactors and lays down a predictive framework for electrified manufacturing.

\section{METHODOLOGY}

Microwave Cavity Description and Temperature Measurements. The microwave cavity used is a metallic cylinder with a $104.92 \mathrm{~mm}$ diameter and $85 \mathrm{~mm}$ height, manufactured at the ITACA Institute at Valencia, ${ }^{26,35,36}$ with a coaxial input port seen in the schematic of Figure 1a. The cavity maximizes the electromagnetic field at the center of the cylinder, in a region of $\sim 10 \mathrm{~mm} \times 15 \mathrm{~mm},{ }^{26,35}$ where the sample [Figure 1b] is placed within a quartz tube (see Figure S1, for further details). The samples are monoliths with channels in the millimeter range [Figure 1c]. The electromagnetic field is created in the range of $2350-2500 \mathrm{MHz}$ by a solid-state generator. A central frequency is identified by the experimental controller based on the coupling resonant frequency to minimize the reflected power back to the input port (determined by the parameter $S_{11}$ ). The bandwidth for the sweep is selected manually, and the controller performs four sweeps over the range of the bandwidth with a fixed power supply of $\sim 130 \mathrm{~W}$. The power deposited in the sample depends on the power supplied and the frequency-dependent port parameter, i.e., $P_{\text {desposited }}(f) \propto P_{\text {input }} \times\left(1-S_{11}(f)\right)$. By operating over a bandwidth of frequencies around the coupling frequency (i.e., the frequency of maximum power deposition and minimum $S_{11}$ ), the power deposited can be controlled (see Figure S2). ${ }^{35}$

A high-fidelity thermal camera (PI 1M, Optris) and an infrared (IR) pyrometer (CTlaser LT, Optris) are attached to the walls of the microwave cavity to measure the lateral side and the quartz reactor wall temperatures, respectively. The thermal camera's working spectral range is from 0.85 to 1.1 $\mu \mathrm{m}$, where the quartz tube has a constant transmission of around $90 \%$. This enables temperature measurements of the sample surface between 450 and $1800{ }^{\circ} \mathrm{C}$. On the other hand, the IR pyrometer has a working spectral range of $8-14 \mu \mathrm{m}$ and measures the quartz wall's temperature between -50 and 975 ${ }^{\circ} \mathrm{C}$. The temperature calibration was done in a conventional electrical furnace with a thermocouple (see Figure S3). The gas-phase temperature downstream of the monolith is measured by placing a thermocouple within a quartz sheath. The gas flows upward from the bottom to allow the placement of the thermocouple. The thermocouple can be translated spatially to obtain an axial profile, enabling comparison with model predictions. Due to the field focus at the central region, the thermocouple does not affect the electromagnetic field, as confirmed by comparing the temperature reading of the thermocouple and IR camera. ${ }^{30}$ Every sample placed in the cavity was cleaned with acetone and calcinated at $700{ }^{\circ} \mathrm{C}$ to eliminate organic impurities before experiments. The flow rate of $\mathrm{N}_{2}$ (Research grade 5.0, Matheson) was controlled through a mass flow controller (GF040, Brooks Instrument).

Multiscale-Multiphysics Model and Parametrization. Modeling of structured reactors in a microwave cavity requires investigation of multiple coupled phenomena: the electromagnetic field and the dissipation of the electromagnetic energy in the solid and the fluid flow through the reactor, and the thermal transport in each phase and between phases. This is achieved using the COMSOL multiphysics software. ${ }^{37}$ The temperature dependence of electrical and thermal transport properties adds additional complexity. For simplicity, temper- 


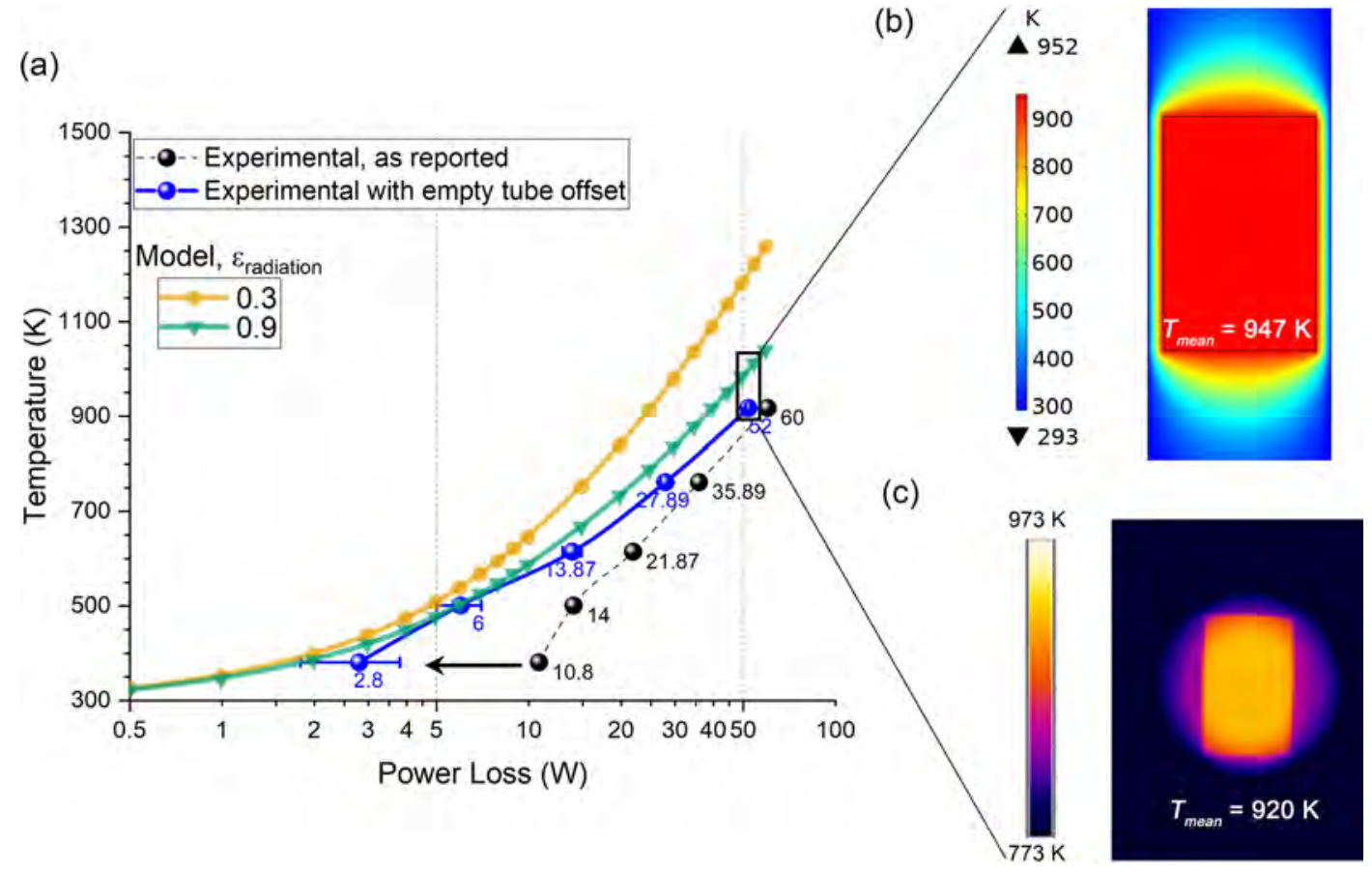

Figure 2. Benchmark experiment (black spheres) of the average solid cylinder temperature vs power absorbed, inferred from the difference between the incident and reflected energy to the microwave coaxial port). Predicted steady-state temperatures (yellow circles and green triangles) for two radiative emissivity values of the silicon carbide cylinder (values indicated in the legend). Offset experimental data by $\sim 8 \pm 1 \mathrm{~W}$ (blue spheres) account for the measured power lost in the cavity without loading the silicon carbide cylinder. Cross-sectional temperature profiles from (b) model and (c) infrared (IR) camera at $\sim 45 \mathrm{~W}$ absorbed power.

ature-independent thermal conductivity and electrical conductivity values are considered (see Figures S4 and S5, for discussion on value selection), and parametric studies are conducted in Impact of Material Properties of Structured Reactors on the Temperature Field. There exist multiple length scales of interest, the length scale of the microwave reactor $(\sim 100 \mathrm{~mm})$, the wavelength of the electromagnetic radiation ( $\sim 122 \mathrm{~mm}$ in free-space), and the size of the monolith $(\sim 10$ $\mathrm{mm})$ and its channels $(\sim 0.1-1 \mathrm{~mm})$; see Figure 1. Such a separation of length scales makes computations challenging. We first consider the steady-state heat transfer equation:

$$
\rho_{j} C_{\mathrm{p}, j} \boldsymbol{u}_{j} \cdot \nabla T_{j}+\nabla \cdot \boldsymbol{q}_{j}=\dot{Q}_{j, \mathrm{emw}}
$$

where,

$$
\boldsymbol{q}_{j}=-\kappa_{j} \nabla T_{j}
$$

is the conductive flux, $\nabla T$ is the temperature gradient, and $\kappa$ is the thermal conductivity of phase $j$ (i.e., solid monolith or fluid inside the channel). The velocity $\boldsymbol{u}$ in eq 1 denotes convective transport in the fluid phase. $\boldsymbol{u}$ is obtained from solving the Navier-Stokes and continuity equations in the laminar flow regime for a fluid of density $\rho$, which varies as a function of temperature (gravity effects are ignored). The magnitude of the electromagnetic energy dissipated per unit volume, i.e., the power loss, $\dot{Q}_{j, \text { emw }}$ in phase $j$ is obtained from the following eq: ${ }^{34,38}$

$$
\dot{Q}_{j, \mathrm{emw}}=0.5 \sigma|\mathbf{E}|^{2}+\pi \epsilon_{\mathrm{c}} \epsilon_{\mathrm{o}} f|\mathbf{E}|^{2}
$$

where $\mathbf{E}$ is the electric field vector, $f$ is the frequency of the microwave radiation, $\epsilon_{\mathrm{o}}$ is the permittivity of vacuum, $\epsilon_{\mathrm{c}}$ is the complex part of the relative permittivity, and $\sigma$ is electrical conductivity. $\mathbf{E}$ is obtained by solving Maxwell's equations using the impedance boundary conditions for the metallic cavity to minimize the computational load. A coaxial port is used to supply the energy to the cavity, and first-order scattering conditions are used at the inlet and outlet of the quartz tube to avoid reflection artifacts. Laminar flow with a defined inlet flow rate and a zero-gauge pressure outlet condition are used. The fluid is at room temperature at the inlet with no flux condition at the outlet. The quartz tube is exposed to room temperature, where it loses heat to the ambient, as described next.

Two additional model parameters are the natural convection coefficient and the radiative emissivity, as the monolith loses heat to surroundings by convection and radiation. The former is obtained from the local Nusselt number, $N u$. The latter is evaluated for a cylinder using the Prandtl number $\mathrm{Pr}$, the cylinder height $H$ and diameter $D$, and the Rayleigh number $\mathrm{R} a$ at the surface ${ }^{39}$

$$
N u_{\mathrm{H}}=\frac{4}{3}\left(\frac{7 R a_{\mathrm{H}} P r}{5(20+21 P r}\right)^{0.25}+\frac{4(272+315 P r) H}{35(64+63 P r) D}
$$

A spectrally average, temperature-independent radiative coefficient of 0.9 for silicon carbide is assumed. ${ }^{40}$ While influencing the temperature field quantitatively, the specific values of these parameters are not expected to modify the overarching findings herein. We discuss the radiative coefficient next. Equations $1-4$ and the rest of the governing equations are implemented using COMSOL's radiofrequency (RF) module, Heat Transfer module, and Laminar Flow module. These modules are solved numerically using the inbuilt finite element method solvers. For the model calibration, steady-state simulations are performed using the Frequency-Stationary study type. A mesh independence-study 
(a)

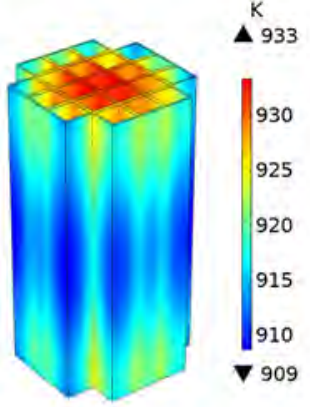

(b)

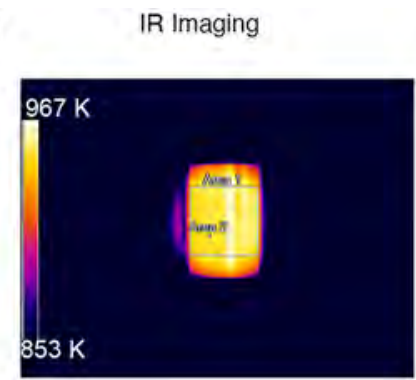

(c)

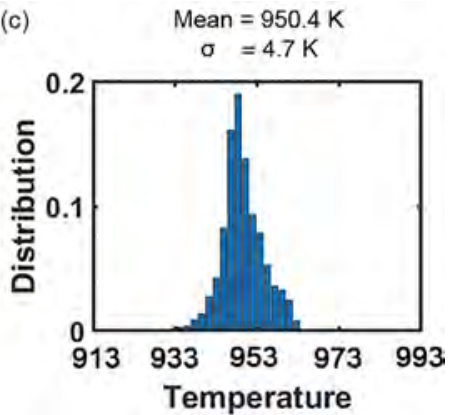

Figure 3. (a) Predicted steady-state temperature in a monolith heated without fluid flow at $2410 \mathrm{MHz}$ with $125 \mathrm{~W}$ input power from the coaxial antenna. (b) Experimental thermal camera image. The pixel distribution in Area 1 rectangle is analyzed in panel $c$ to extract temperature distribution.

was conducted, and owing to the small size of the monolith's walls, a COMSOL inbuilt "Extremely Fine" mesh was utilized to ensure at least 6 mesh elements per wavelength $(\sim 122 \mathrm{~mm}$ in air) in the domain. The mesh sizes of other domains, including the microwave cavity and the monolith channels, were varied. For higher accuracy, inbuilt "Extremely Fine" settings were selected despite a $<5 \%$ difference between the power absorbed of "Finer" and "Extremely Fine" sizes. This expensive meshing procedure provided high fidelity results. Eight cores (Intel Xeon CPUs E5-2695, 192 GB RAM) were utilized in solving the multiphysics model.

\section{RESULTS AND DISCUSSION}

Model Assessment Against Experiments under Static (No Flow) Conditions. A simplifying modeling procedure vis-à-vis the operation of the electromagnetic generator is utilized. The minimum coupling frequency is identified by sweeping over the possible operating frequencies. Simulations are then run at this fixed frequency at varying power input. In the benchmarking shown in Figure 2, we employ a more complex system of a silicon carbide cylinder $(D=10 \mathrm{~mm}, H=$ $15 \mathrm{~mm}$ ). The experiments (black spheres) are conducted over different bandwidths, and the computations are performed at different powers, both of which translate into varying the power absorbed by the cylinder ( $x$-axis). The experiments reported an absorbed power of 7-11 W at $20 \mathrm{MHz}$ bandwidth when the quartz tube is free of any microwave absorbing sample (i.e., an empty tube). Since metals have a finite electrical conductivity, microwaves can penetrate a few microns and dissipate heat in the cavity walls. Furthermore, possible leaks may exist in a real system. The exact modeling of losses inside the metallic cavity boundary is challenging. For instance, the surface roughness of the metal can affect the ratio of the dissipated power in the sample and the walls. Moreover, losses in the coupling network (the input antenna and coupling adjustment subsystem) are ignored. Here we find that offsetting the experimental absorbed power values by an absolute power value $(8 \pm 1 \mathrm{~W}$ in the case of the solid cylinder) allows for the predicted temperature field [Figure $2 \mathrm{~b}$ ] to be in good agreement with these offset IR camera data [Figure 2c]. Our calculations indicate that describing the experimental data by varying the input power instead of the bandwidth is a tractable multiscale-multiphysics modeling approach. Radiation plays a role at higher temperatures, as expected; an average radiative coefficient of $\sim 0.9$ is reasonable for nearly quantitative agreement with experimental data (see typical results in Figure 2a). Given how difficult it is to predict the reactor temperature, we deem this satisfactory and do not seek a more precise value.

Next, we compare the predicted steady-state temperature in a silicon carbide monolith without flow against experimental IR camera measurements. A silicon carbide honeycomb monolith with 21 square channels of $1.3 \mathrm{~mm} \times 1.3 \mathrm{~mm}$, shown in Figure 3a, is placed in a quartz tube with $10 \mathrm{~mm}$ ID (internal diameter). The monolith, cut from a commercial diesel particle filter (DPF, Ibiden, USA) with a diamondcoated blade, is made of $\alpha$-SiC with a cell density of 300 cells per square inch. Its length of $15 \mathrm{~mm}$ enables maximum overlap with the electromagnetic field in the microwave cavity. We find that the predicted steady-state temperature $(T \sim 915 \mathrm{~K})$ is in excellent agreement ( $<5 \%$ difference) with the measurements$(T \sim 950 \mathrm{~K})$. The experimental temperature of monolith walls varies between 933 and $963 \mathrm{~K}$, with a mean of $950.4 \mathrm{~K}$ and a standard deviation of $4.7 \mathrm{~K}$ (square area (Area 1) in Figure $3 \mathrm{~b})$. The predicted three-dimensional temperature field shows a radial inhomogeneity as the monolith's side edges lose heat, leading to higher temperatures in the middle, exhibiting a similar $30 \mathrm{~K}$ spread.

Experimental and Computational Steady-State Temperature Profiles under Flow. The temperature difference between the structured reactor walls and the fluid phase has been postulated as a critical factor in energy savings and suppressing unselective gas-phase chemistry. Prior work reported a temperature gap of $20-50 \mathrm{~K}$ for a dilute stream of alkane. ${ }^{10,30}$ To understand the temperature field, we perform flow simulations and experiments in a silicon carbide monolith (Figure 4). Air enters the quartz tube (denoted by the arrow at $0 \mathrm{~mm}$ ) at 100 standard cubic centimeters per minute (SCCM) at room temperature and atmospheric pressure and flows through the monolith channels. The monolith is heated by absorbing the microwave radiation and transfers energy to the flowing air via conduction and convection. In Figure $4 \mathrm{~b}$, we plot the predicted temperature along the centerline of the central channel of the monolith (blue line) in the gas phase and the solid of this channel (blue triangles). At this flow rate, the Reynolds number at the maximum gas temperature (i.e., $\sim 900 \mathrm{~K}$ ) is $R e=3.6$ supporting the laminar flow assumption. The red data depicts the monolith IR-measured temperature from pixel analysis, and the black spheres are the gas temperature downstream of the monolith measured using a sheathed thermocouple.

The model matches the average experimental wall temperature accounting for the empty tube losses presented above. The experimental measurements agree with the computations 
(a)

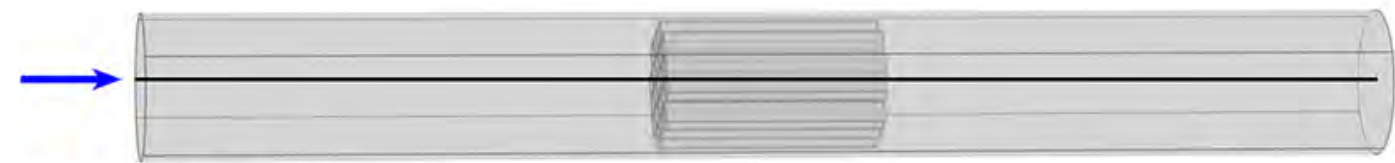

Inlet, Location $=0 \mathrm{~mm}$

Location $=85 \mathrm{~mm}$

(b)

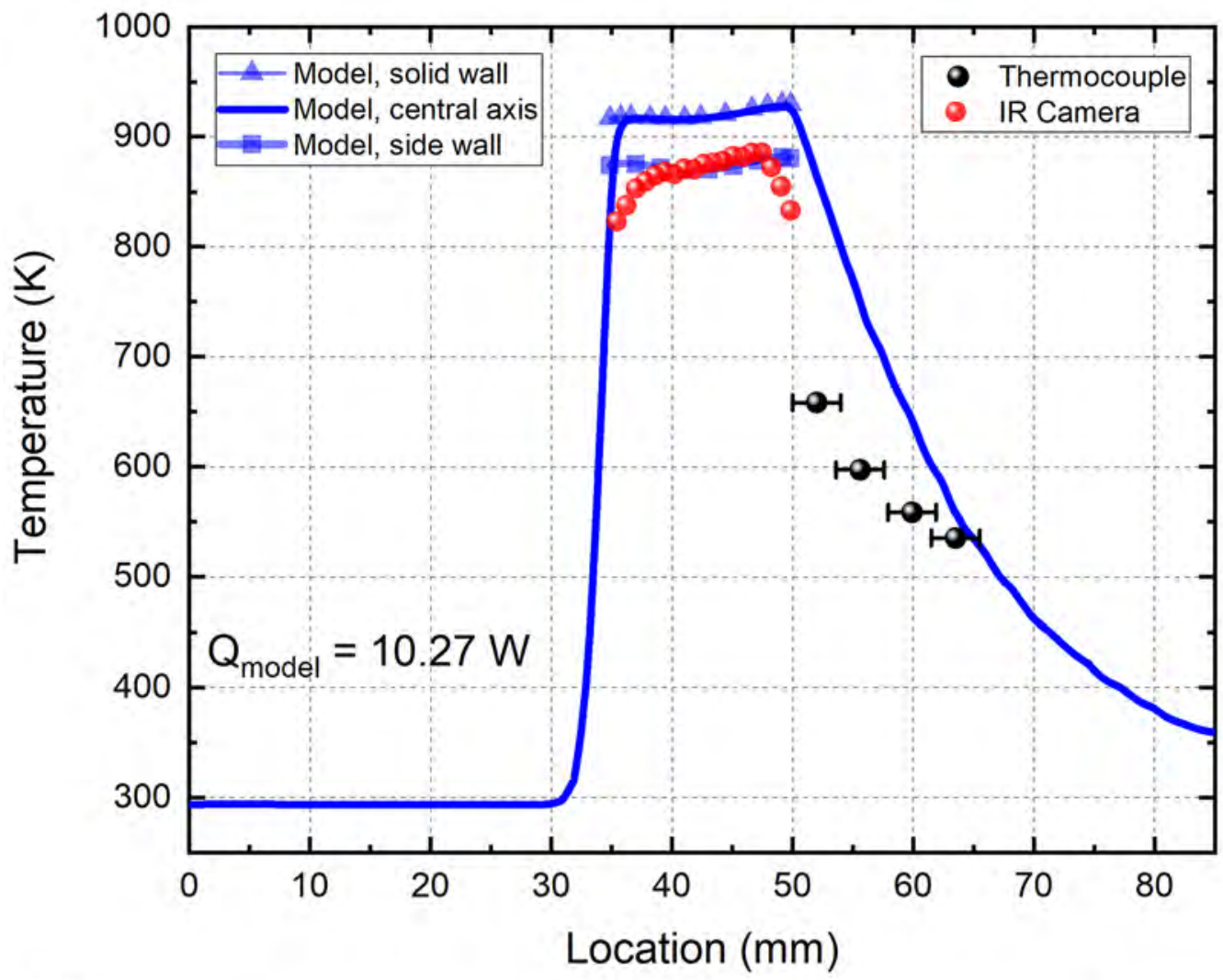

(c)

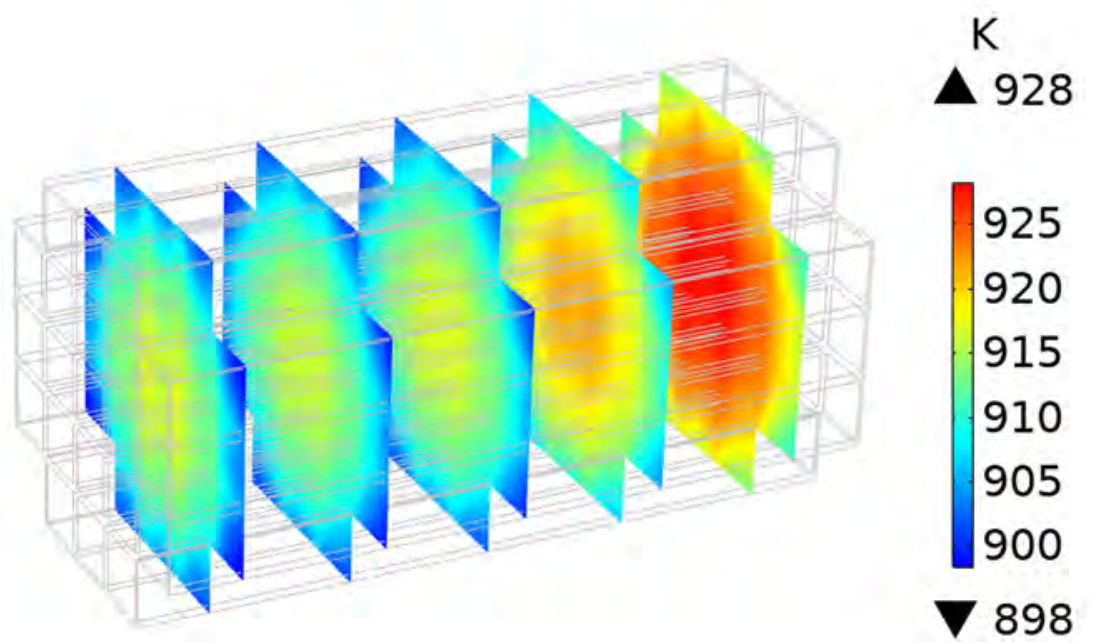

Figure 4. (a) Geometry of the quartz tube with the monolith placed in the middle and air flowing from the left (arrow) at 100 SCCM. The axis of tube symmetry is marked in black. The blue triangles denote the predicted temperature of the solid wall around the central channel, and blue squares are the temperatures of the outer edge of the side channel. The red and black points show the temperatures measured using an IR camera and thermocouple, respectively. Error bars account for the thermocouple placement error $( \pm 2 \mathrm{~mm})$ downstream from the monolith. (c) Crosssectional color maps of temperature in the monolith at $z=1.5,4.5,7.5,10.5$, and $13.5 \mathrm{~mm}$ from the monolith inlet. The temperature is radially symmetric and reduces outward from the quartz tube due to heat loss. 
(a)

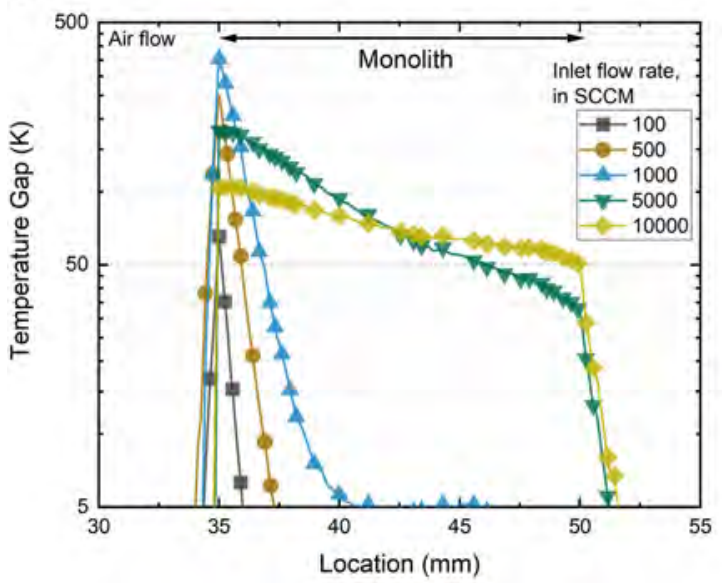

(b)

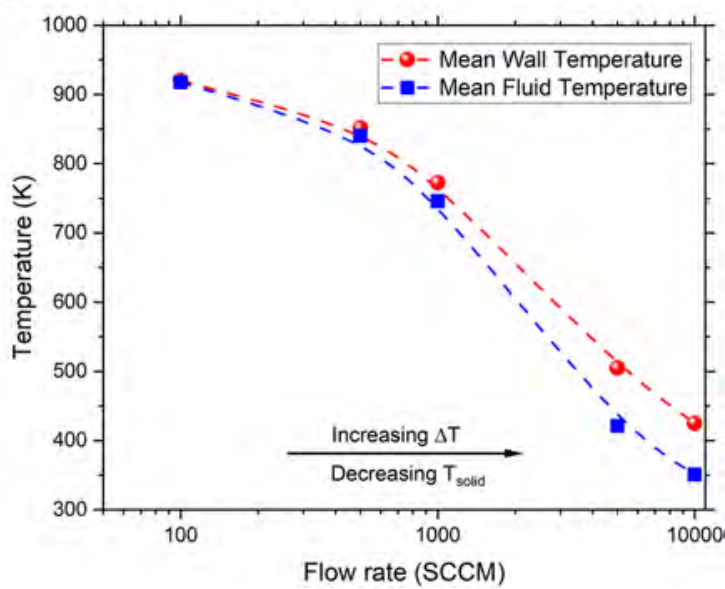

Figure 5. (a) Predicted temperature difference between the SiC monolith walls and the gas in the center of the middle channel and (b) average wall and fluid temperature in the middle channel as a function of inlet air flow. The monolith extends from 35 to $50 \mathrm{~mm}$ in panel a. The maximum flow rate considered corresponds to a laminar flow of $R e \sim 2100$.
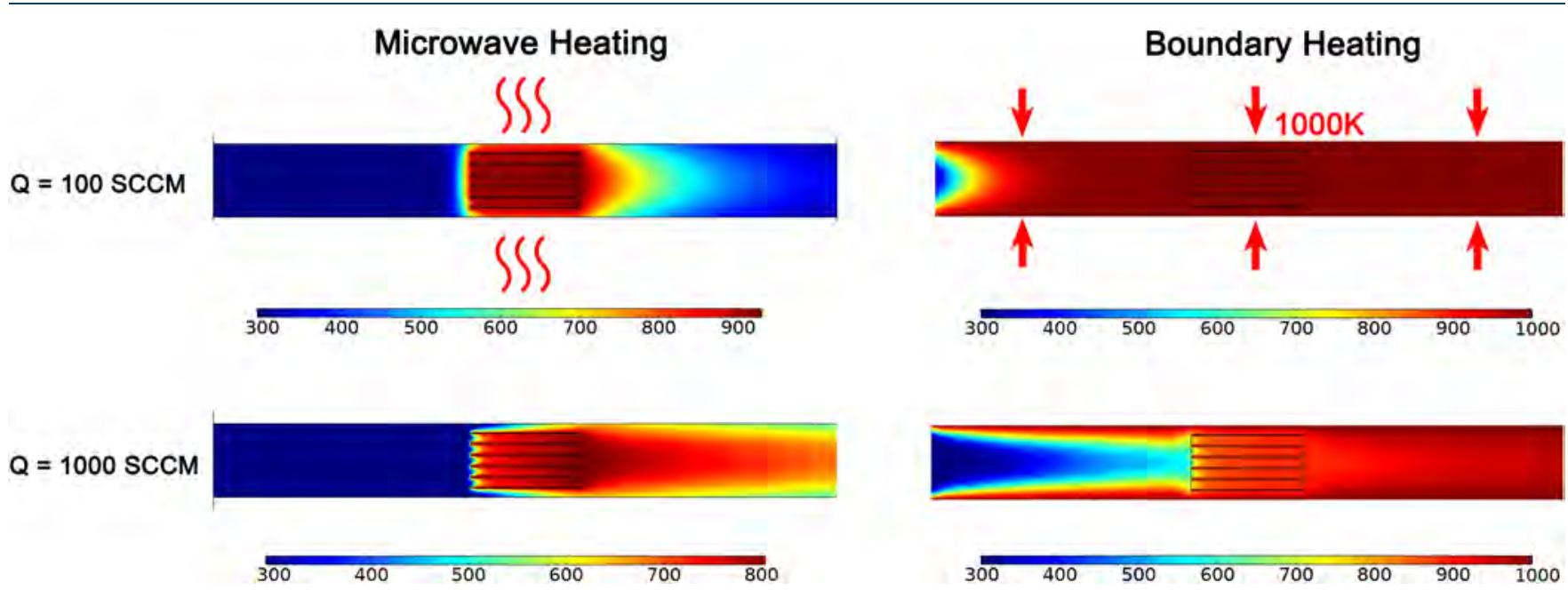

Figure 6. Cross section of the temperature field in a $85 \mathrm{~mm}$ long quartz tube with the monolith placed at the center with air flowing at two different flow rates. (Left) Heated with microwaves with a (right) quartz boundary maintained at $1000 \mathrm{~K}$. Temperature bars are in Kelvin.

in the center of the solid within a few degrees, whereas, at the edges of the monolith, the temperature drops possibly due to the existence of finite IR camera window size, causing edge effects. The experimental-model agreement is reasonable and, combined with the comparisons under static conditions discussed above, supports modeling as an in situ tool to infer temperature gradients inside the monolith channels.

The model predicts a nearly isothermal solid due to silicon carbide's high thermal conductivity $(\sim 50-100 \mathrm{~W} / \mathrm{m} \mathrm{K}$ at 900 $\mathrm{K}) .{ }^{41}$ At the monolith's inlet, the air temperature is lower than that of the wall by $\sim 50 \mathrm{~K}$. Within $\sim 2 \mathrm{~mm}$ from the entrance, the air temperature rises and differs from the wall temperature $<5 \mathrm{~K}$ for the remainder of the monolith. The steady-state temperature cross sections, shown in Figure 4c, indicate thermal equilibration between the walls and the air in each channel due to fast radial heat transfer. A small overall temperature drop of $30 \mathrm{~K}(\sim 3 \%)$ occurs in the gas temperature in the radial direction due to outward heat losses. The temperature of the monolith side wall in Figure $4 \mathrm{~b}$ (blue squares) is in closer agreement to the experimental temperature, as the IR image is from a line that runs along the outside of the monolith instead of the central axis. However, for practical purposes, the monolith and gases in contact may be considered isothermal. Under these conditions, even though selective heating occurs, the gas equilibrates thermally with the solid within the monolith rapidly, and no transverse (perpendicular to the flow) temperature gradient exists. While approaching steady-state (i.e., under transient conditions), we find that gas and solid temperatures evolve under a similar ramping rate. We highlight that our calculations do not consider chemical reactions (beyond this work scope), whose endothermicity can increase the temperature difference.

Interestingly, both experiments and the model show a rapid temperature drop off by $80-170 \mathrm{~K}$ past the heated monolith within 2-5 $\mathrm{mm}$ from the monolith edge. This finding is consistent with literature findings where a $150 \mathrm{~K}$ drop off was measured $2 \mathrm{~mm}$ past a monolith. ${ }^{30}$ The rapid rise of air temperature at the entrance and the drop-off post monolith are a consequence of its low volumetric heat capacity, i.e., density $\rho \times$ specific heat capacity $C_{\mathrm{p}}$. We return to this point in more detail below.

The flow rate is expected to play a role in the temperature gradient as it affects the residence time and the energy removed from the solid. We predict the temperature difference 

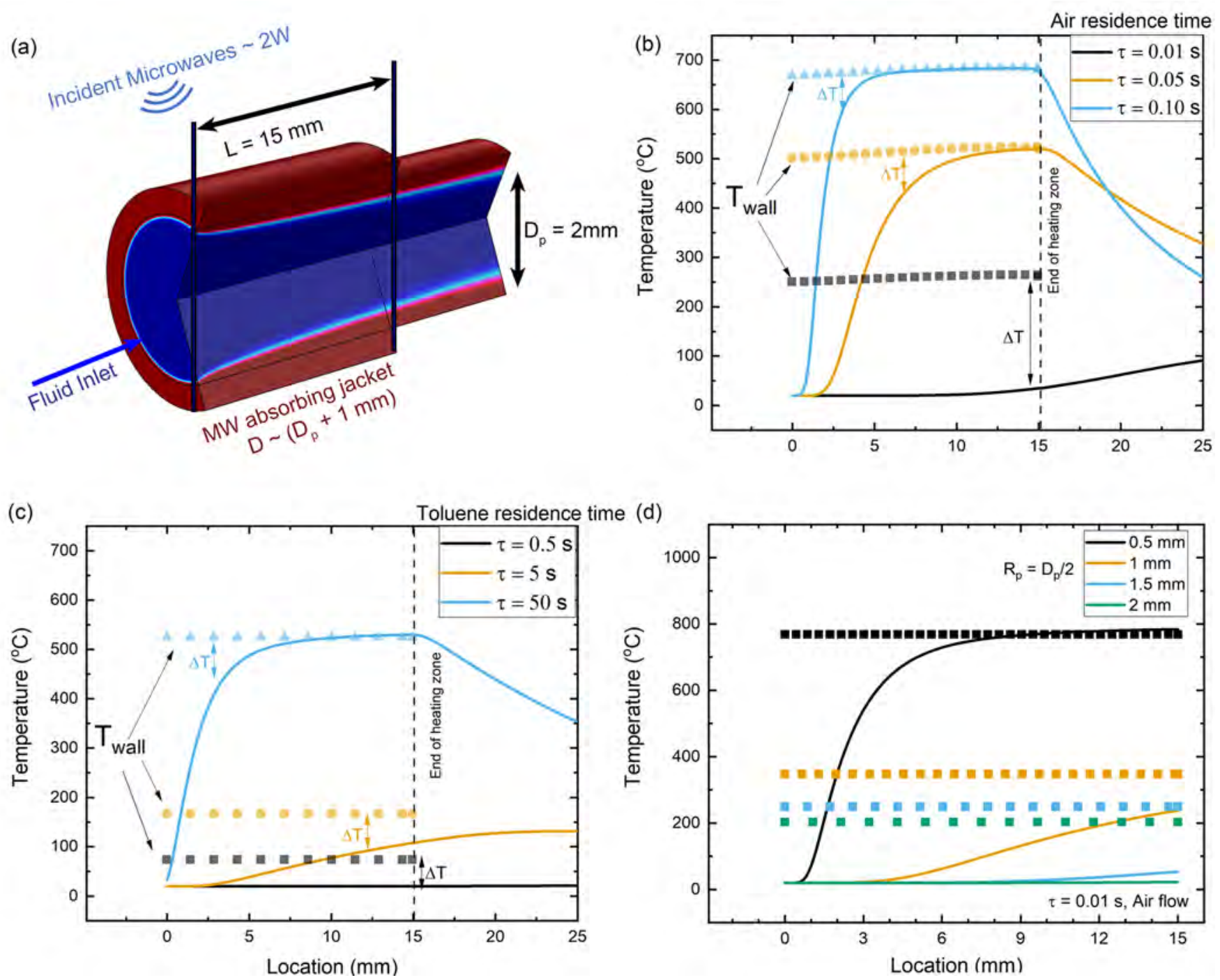

Figure 7. (a) Geometry for COMSOL simulations of a single flow channel surrounded by a $0.5 \mathrm{~mm}$ thick solid material and $15 \mathrm{~mm}$ length. Two W power is uniformly dissipated into the solid. Axial temperature profiles at the center of the channel (solid lines) and the solid wall (points) for different residence times for the flow of (b) air and (c) toluene. (d) Role of channel radius in the evolution of fluid temperature and wall temperature shown for the flow of air at $0.01 \mathrm{~s}$ residence time.

between the wall and the flowing air at the center of the monolith channel for different airflow rates [Figure 5a]. For inlet flow rates lower than $1000 \operatorname{SCCM}(R e=36$ at $900 \mathrm{~K})$, corresponding to a superficial residence time of $0.07 \mathrm{~s}$ for a 15 $\mathrm{mm}$ long monolith and the specific channel size, the temperature gap becomes small $(<5 \mathrm{~K})$ within a few $\mathrm{mm}$ from the entrance. The walls and the fluid equilibrate thermally due to the low volumetric heat capacity of air and the small radius of the monolith channel, allowing rapid heat transfer from the wall to the gas. For higher flow rates (a residence time $<0.01 \mathrm{~s}$ ), a measurable temperature gap ( $\sim 50 \mathrm{~K})$ occurs over most of the monolith. As the flow rate increases, the average wall temperature is lower [Figure $5 b$ ] due to more energy removed by the fluid. We analyze both these findings with a simplified model of a single channel in the next section.

Significantly, these results indicate that the thermal transport between walls and gas-phase minimizes the temperature gradients under typical laboratory flow conditions $(\sim 100$ SCCM). Therefore, despite selective microwave heating of a solid structured bed, the difference in temperatures and the subsequent suppression of gas-phase chemistry may be insignificant under many conditions. Instead, the monolith's localized heating, followed by the rapid quenching of the gas past the monolith exit, may be responsible for suppressing undesirable side reactions. Such rapid quenching is unattainable in conventional heating in typical laboratory furnaces where the quartz tube's whole length is heated (i.e., even downstream of the monolith). To further assess this hypothesis, we set a temperature along the quartz wall boundary $(\sim 1000 \mathrm{~K})$ to mimic a conventionally heated system and evaluate the temperature field developed in the quartz tube in Figure 6. Heating in microwaves is spatially localized to the solid structure.

In contrast, boundary heating allows air to heat throughout the reactor, especially at low flow rates (100 SCCM). At larger flow rates (1000 SCCM), the region downstream from the microwave-heated monolith can also be hot as heat is convected axially. However, the air is cold upstream of the monolith, allowing suppression of gas-phase chemistry compared to a furnace-heated system.

Effects of Fluid Properties and Channel Diameter of an MW-Heated Single Channel. To get deeper insights, we 
Table 1. Order of Magnitude $(O)$ of Properties for Air and Toluene at Room Temperature, Corresponding Heat Transfer Coefficient Determined Using Eq 9, and Thermal Time Constant for a Channel $0.5 \mathrm{~mm}$ in Size

\begin{tabular}{llccccc}
\multicolumn{1}{c}{ fluid } & $\rho\left[\mathrm{kg} / \mathrm{m}^{3}\right]$ & $\mathrm{C}_{\mathrm{p}}[\mathrm{J} / \mathrm{kg} / \mathrm{K}]$ & $\mu[\mathrm{Pa} \mathrm{s}]$ & $k[\mathrm{~W} / \mathrm{m} / \mathrm{K}]$ & $h\left[\mathrm{~W} / \mathrm{m}^{2} / \mathrm{K}\right]($ from eq 9) & thermal time constant $\tau_{\text {eq }}[\mathrm{s}]($ from eq 8$)$ \\
air & $O(1)$ & $O\left(10^{3}\right)$ & $O\left(10^{-5}\right)$ & $O\left(10^{-2}\right)$ & $O\left(10^{-2}\right)$ & $\sim 0.025$ \\
toluene & $O\left(10^{3}\right)$ & $O\left(10^{3}\right)$ & $O\left(10^{-3}\right)$ & $O\left(10^{-1}\right)$ & $O\left(10^{-1}\right)$ & $\sim 2.5$ \\
\hline
\end{tabular}

focus on a single cylindrical channel with walls made of $\mathrm{SiC}$, as shown in Figure 7a. Microwave power $\dot{Q}_{\mathrm{mw}}$ of $2 \mathrm{~W}$ is deposited into the solid and the heat transfer coefficient from the solid to the ambient is $15 \mathrm{~W} / \mathrm{m}^{2} \mathrm{~K}$ (radiative losses are omitted for simplicity as they do not alter the qualitative findings). To eliminate complexity arising from hydrodynamics, a fully developed flow through the channel is considered. We plot the axial temperature profile at the center of the channel through which air flows at varying residence times (or flow rates) as well as the average wall temperature in Figure $7 \mathrm{~b}$. The wall temperature changes only slightly through the heating zone due to the high wall thermal conductivity in line with findings in a monolith discussed above. The air temperature for residence times $\tau \geq 0.05 \mathrm{~s}$ rises rapidly to the wall temperature. For shorter residence times, e.g., $0.01 \mathrm{~s}$, the flow is fast, resulting in minimal temperature rise (consistent with eq 11 , see discussion in An Analytical Criterion of Evaluation of the Fluid-Solid Temperature Difference). Additionally, the lower wall temperature at higher flow rates results from the more considerable energy carried away by the flowing gas.

To quantify the fluid's role, we consider the flow of a nonpolar liquid, like toluene, through the channel in Figure $7 \mathrm{c}$. A nonpolar liquid allows us to ignore microwaves' direct dissipation in the liquid phase to maximize selective heating. The highest temperature shown is beyond typical laboratory experiments, but we include it to strengthen the insights. In this system, we find that far slower flows $(\tau \sim 5 \mathrm{~s})$ can sustain a temperature gap as the liquid toluene has a higher density $\rho \times$ specific heat capacity $C_{p}$ value than air. This indicates that the selection of the fluid itself can tailor the solid-fluid temperature gap. Finally, to understand the impact of channel size on the temperature gap, we consider different channel radii in Figure $7 \mathrm{~d}$ and find that, for the same amount of deposited power, the fluid and wall temperatures in larger channels are lower and the temperature gap is larger.

An Analytical Criterion of Evaluation of the FluidSolid Temperature Difference. We propose a simple, lumped analytical model for the mean temperature of a fluid flowing through a cylindrical channel to provide intuitive insights. We assume that the rate of temperature rise in the wall is much higher compared to heat loss to the surroundings and the fluid, thereby allowing the solid phase to reach a quasisteady temperature, $T_{s}$. This assumption relies on the rapid deposition of the microwave power, as the incident photons travel in the vacuum at the speed of light, and the subsequent rapid thermal equilibration of the solid due to its high thermal conduction. Energy from the walls is conducted and convected to the fluid. We estimate the evolution of the fluid temperature, $T$, from a simple energy balance over a differential cylindrical volume:

$$
\rho C_{\mathrm{p}} u\left(\pi R^{2}\right)\left(T_{@ z}-T_{@ z+\Delta z}\right)+h(2 \pi R \Delta z)\left(T_{\mathrm{s}}-T_{@ z}\right)=0
$$

Here $R$ is the channel's radius, and $h$ is the heat transfer coefficient between the solid and the gas obtained from Nusselt number, $\mathrm{Nu}$, correlations. This simple model of the classical Graetz heating lumps all radial effects into an effective heat transfer coefficient. The temperature evolution in the gas, with the fluid entering at temperature $T_{\mathrm{o}}$ (at $z=0$ ), for plug flow of fluid of velocity $\boldsymbol{u}$ is

$$
\begin{aligned}
& T(z)=T_{\mathrm{s}}-\left(T_{\mathrm{s}}-T_{\mathrm{o}}\right) \exp (-z / \zeta) \\
& \zeta=\frac{\rho C_{\mathrm{p}} \boldsymbol{u} R}{2 h}
\end{aligned}
$$

Applying a space-time transformation of the axial coordinate, $z$ $=\boldsymbol{u}$, we obtain the thermal time constant $\tau_{\mathrm{eq}}$ :

$$
\tau_{\text {eq }}=\frac{\rho C_{\mathrm{p}} R}{2 h}=\frac{\rho C_{\mathrm{p}} R^{2}}{N u_{\mathrm{R}} k}=\frac{R^{2}}{N u_{\mathrm{R}} \alpha}
$$

where $k$ is the thermal conductivity of the fluid, $\alpha$ is the thermal diffusivity, and $N u_{\mathrm{R}}$ is the Nusselt number, with the channel radius as the characteristic length scale (see eq 9). The normalized temperature difference evolves as $\frac{T_{\mathrm{s}}-T(z)}{T_{\mathrm{s}}-T_{\mathrm{o}}}=\exp \left(-t / \tau_{\mathrm{eq}}\right)$.

Furthermore, we consider a fully developed laminar flow through a cylindrical channel, where the average $N u_{\mathrm{R}}$ is known to be $\mathrm{be}^{42}$

$$
N u_{\mathrm{R}}=\frac{2 h R}{k}=3.66
$$

This value of the $N u_{\mathrm{R}}$ number provides an upper bound for the thermal time constant as, during the evolution of the hydrodynamic flow profile (developing flow), the local values for $N u_{\mathrm{R}}$ can be much higher. ${ }^{42}$

The gas reaches the wall temperature theoretically at infinite length and practically within $4-6 \zeta$ or within $4-6$ thermaltime constants. When the residence time $\tau$ is less than or in the same order as the thermal time constant, $\tau_{\text {eq }}$, the fluid does not have sufficient time to heat, and a solid-fluid temperature gap occurs. The transverse heat transfer time scale depends quadratically on the channel radius and inversely on the fluid thermal diffusivity. For example, when the radius varies from $0.5 \mathrm{~mm}$ to $2 \mathrm{~mm}$, the thermal equilibration time increases 16 times. Common gases reach thermal equilibrium at similar times, given their close thermal diffusivity. Liquids, on the other hand, have lower thermal diffusivity, and thus, equilibrate thermally more slowly. To assess the thermal time, we consider a single cylindrical channel of a $0.5 \mathrm{~mm}$ radius and $15 \mathrm{~mm}$ in length to imitate our experimental dimensions and two fluids, air and toluene. Both fluids are microwave transparent and are heated from the walls. The analysis in Table 1 shows that despite possessing a larger thermal conductivity and heat transfer coefficient, toluene can sustain a wall-fluid temperature difference for longer residence times. There may be practical limits to developing a large temperature difference in liquids as their flow rates are often slower than those of gases. In contrast, a temperature difference for gases exists for sufficiently fast flows only, owing to their larger thermal 
(a)

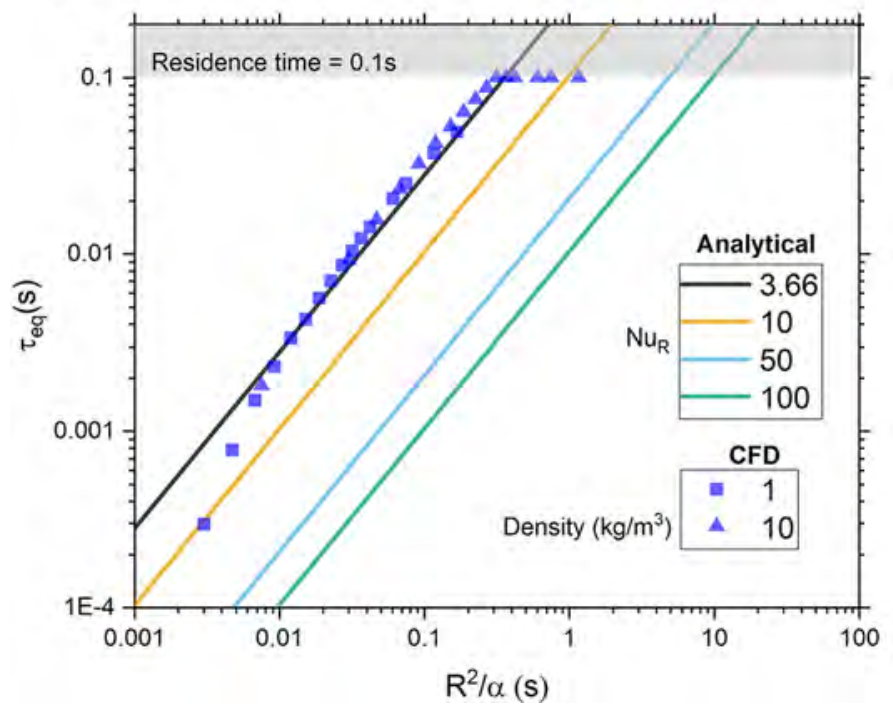

(b)

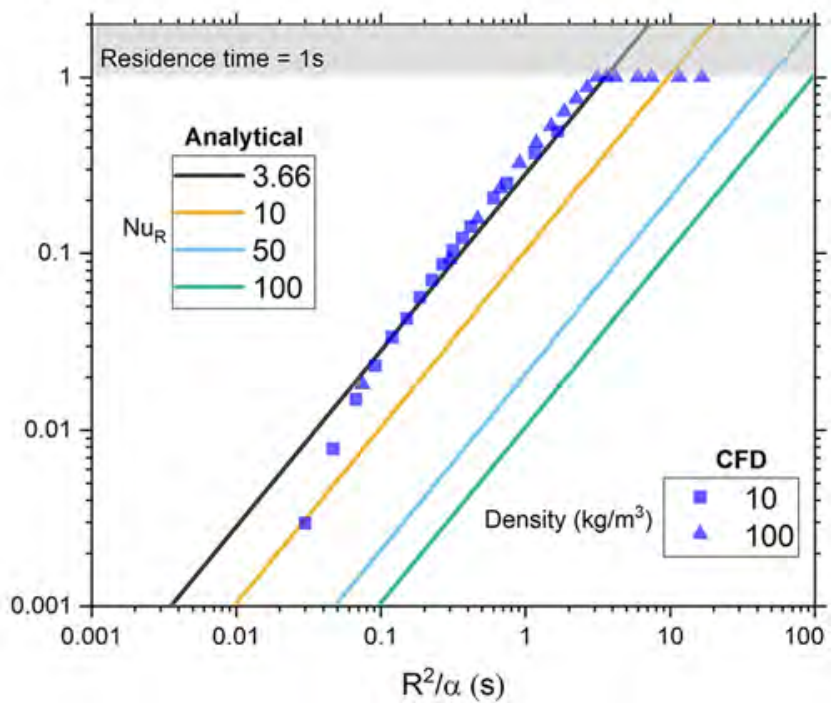

Figure 8. Thermal time constant obtained from CFD simulations (scatter points) vs simple analytical model for different Nusselt numbers (lines) at a residence time of (a) $0.1 \mathrm{~s}$ and (b) $1 \mathrm{~s}$. Different fluid densities are modeled in CFD (squares and triangles).

diffusivity. These findings are in good qualitative agreement with the monolith results above.

To assess the simple analytical model, we compare results from eqs 7 and 8 to CFD data. In the latter, the temperature of the wall $T_{s}$ evolves under microwave heating, and the fluid entrance temperature is $20{ }^{\circ} \mathrm{C}$. The thermal time constant is obtained by dividing the length at which the temperature at the center of the channel becomes equal to $67 \%$ of the average wall temperature by the flow velocity. Fluids of two densities at two different residence times are considered (Figure 8). The CFDbased thermal time-constant vs $R^{2} / \alpha$ agrees excellently with eq 8 along the $N u_{\mathrm{R}}=3.66$ line. The CFD simulations have an upper bound due to the thermal time constant not exceeding the residence time (shown by the horizontal distribution of scatter points). The analysis allows us to conclude that no temperature gap exists in monoliths when the thermal time constant is too short $\left(\tau_{\text {eq }} \ll \tau\right)$. The former $\left(\tau_{\text {eq }}\right)$ is controlled mainly by the fluid thermal diffusivity $\left(\kappa / \rho C_{\mathrm{p}}\right)$ and the square of the channel radius $R^{2}$.The choice of the fluid is often dictated by the catalyst activity and selectivity and the relative volatilities of reactants and products. It will be considerably different for gas and liquid phase reactors. We propose that the easier parameter to engineer the thermal equilibrium time is through the channel diameter. The latter $(\tau)$ is controlled by the flow rate and the reactor length; both are amenable to tuning, but they also affect the conversion and the catalyst amount loaded. Lastly, the interplay between $\tau_{\text {eq }}$ and $\tau$ may allow for tuning the temperature gap between the solid and fluid phase under the cyclical power supply, i.e., cycles of power on/off, beyond the constant power case studied here. This is worth studying in future work.

In the above-simplified model, we assumed a constant wall temperature. The CFD simulations for $\mathrm{SiC}$ support a small variation in axial wall temperature. This can be rationalized from the Biot number, $B i_{\mathrm{z}}=\frac{h L}{\kappa}$, which for a solid of high thermal conductivity, such as $\mathrm{SiC}$, of $15 \mathrm{~mm}$ length, is very low $\left(B i_{\mathrm{z}}<10^{-3}\right)$.
An overall steady-state energy balance including the fluid and the solid reveals the role of material properties and flow rate in temperature:

$$
\begin{aligned}
& \rho C_{\mathrm{p}} u\left(\pi R^{2}\right)\left(T_{\mathrm{o}}-T_{\mathrm{f}}\right)+\rho_{\mathrm{s}} C_{\mathrm{s}, \mathrm{p}} V_{\mathrm{s}}\left(T_{\mathrm{o}, \mathrm{s}}-T_{\mathrm{s}}\right)+\dot{Q}_{\mathrm{mw}}-\dot{Q}_{\text {surroundings }}=0 \\
& T_{\mathrm{f}}=T_{\mathrm{o}}+\frac{\dot{Q}_{\mathrm{mw}}-\dot{Q}_{\text {surroundings }}}{\rho C_{\mathrm{p}} u\left(\pi R^{2}\right)}=T_{\mathrm{o}}+\frac{\left(\dot{Q}_{\mathrm{mw}}-\dot{Q}_{\text {surroundings }}\right) \tau}{\rho C_{\mathrm{p}} V_{f}}
\end{aligned}
$$

where $V_{\mathrm{f}}$ is the volume of the fluid, i.e., the channel volume of the structured bed and $\dot{Q}_{\text {surroundings }}$ is the heat lost to the ambient via convection and radiation. Here $\dot{Q}_{\mathrm{mw}}$ is the microwave energy absorbed by the wall. The second term of eq 10 drops off for an isothermal solid. This overall balance predicts that considerably more power will be needed for heating liquids than gases to reach the same temperature due to the difference in density. Furthermore, a higher flow rate (a lower residence time) leads to a lower temperature for the same power supply as there is less time to transfer heat from the wall to the solid. These insights are intuitive and consistent with Figure 7.

Impact of Material Properties of Structured Reactors on the Temperature Field. In the above discussion, the power deposited into the solid $\dot{Q}_{\mathrm{mw}}$ depends on the available microwave absorbing material volume $V_{\text {emw }}$, the incident power per unit volume $\dot{Q}_{\mathrm{emw}}$, the developed electric field $\mathbf{E}$ in the material at frequency $f(\mathrm{rad} / \mathrm{s})$ and the material properties, including the electrical conductivity $\sigma$ and the complex part of the relative permittivity $\epsilon_{\mathrm{c}}$

$$
\dot{Q}_{\mathrm{mw}}=\dot{Q}_{\mathrm{emw}} V_{\mathrm{emw}} \sim V_{\mathrm{emw}}\left(0.5 \sigma|\mathbf{E}|^{2}+\pi \epsilon_{\mathrm{c}} \epsilon_{\mathrm{o}} f|\mathbf{E}|^{2}\right)
$$

where $\epsilon_{\mathrm{o}}$ is the permittivity of the free space. The total power absorbed by the microwave absorbing material is affected by the electrical conductivity (i.e., the first term in eq 12) and the complex permittivity (i.e., the second term). The relative contribution of the two mechanisms at $2.45 \mathrm{GHz}$ frequency, for sufficiently long penetration depth, is approximated by 


\section{Silicon Carbide}

(a)

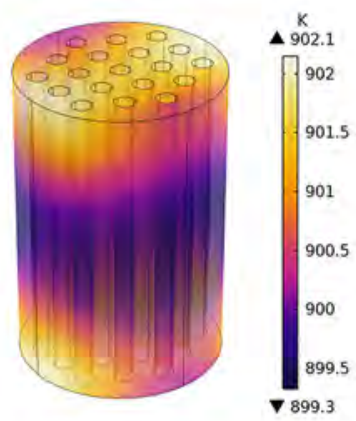

(b)

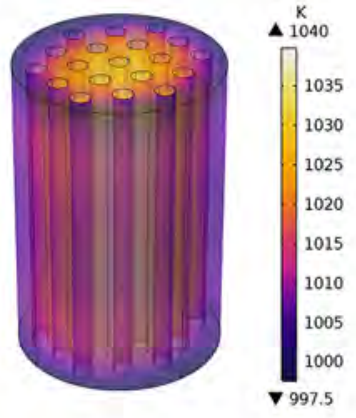

'Electrically inhibited' Silicon Carbide

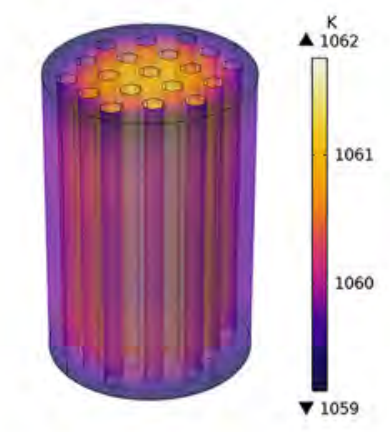

(c) (d)

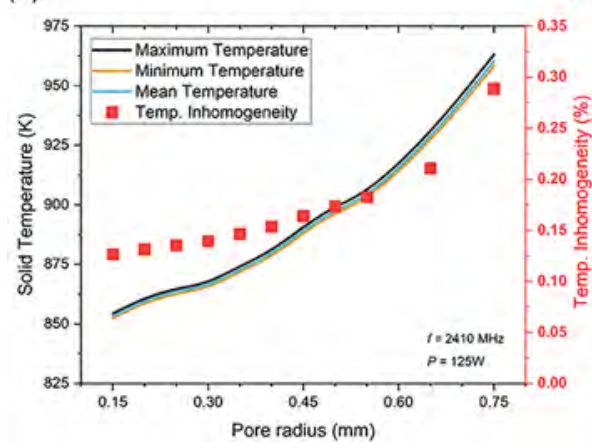

(f)

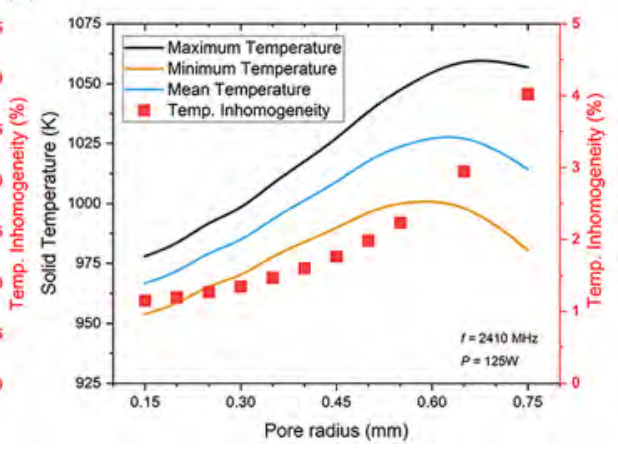

(e)

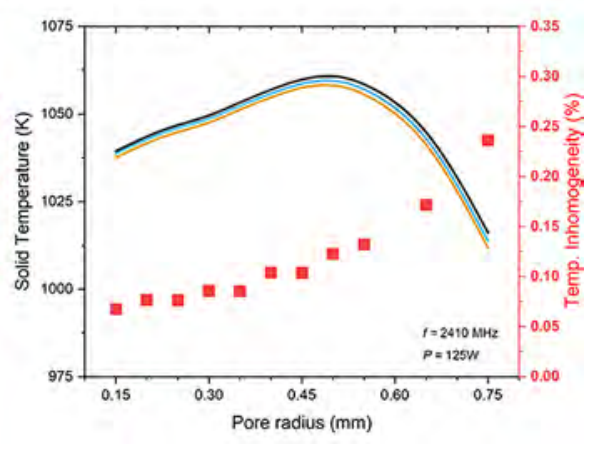

Figure 9. Role of thermal conductivity $\kappa(\mathrm{W} / \mathrm{m} \mathrm{K})$ and electrical conductivity $\sigma(\mathrm{S} / \mathrm{m})$ in the heating of structured beds at a constant frequency of $2410 \mathrm{MHz}$. The different $\{\kappa, \sigma\}$ values are (a) $\{100,50\}$ for silicon carbide, (b) $\{5,5\}$ for iron oxide, and (c) $\{100,5\}$ for an insulating silicon carbide-like material. The temperature maps $(\mathrm{a}-\mathrm{c})$ are shown for a channel radius of $0.5 \mathrm{~mm}$. (d-f) Corresponding temperatures $($ left-y) for each case as a function of channel size. Note the difference in scale across all figures.

$$
\frac{\dot{Q}_{\text {conduction }}}{\dot{Q}_{\text {dielectric }}} \sim 7.3 \frac{\sigma}{\epsilon_{c}}
$$

where the electrical conductivity $\sigma$ is in units of $\mathrm{S} / \mathrm{m}$. For heating of liquids, such as ethanol, the dielectric losses $(\sigma \sim$ $10^{-3}, \epsilon_{\mathrm{c}} \sim 5$ at room temperature and $\left.2.45 \mathrm{GHz}^{43}\right)$ dominate, whereas, for solids, such as silicon carbide $\left(\sigma \sim 0.1-100, \epsilon_{\mathrm{c}} \sim\right.$ 2 ), conduction losses become significant. The addition of ionic salts to liquids can enhance conduction losses and microwave heating. ${ }^{33}$ Similarly, the inclusion of impurities and nanostructures can tailor the electrical conductivity and dielectric properties of silicon carbide. ${ }^{13}$ We analyze the role of these properties along with thermal conductivity next.

Heating structured beds by microwaves entail (a) absorption and conversion of electromagnetic energy to thermal energy and (b) thermal transport within the structure to attain thermal equilibrium. The relative permittivity and the electrical conductivity $\sigma$ of materials control the first step's efficacy; the thermal conductivity $\kappa$ determines the second step. To quantitatively understand the material properties' role, we consider three morphologically identical structures in Figure 9. These beds have an external diameter of $10 \mathrm{~mm}$ and length of $15 \mathrm{~mm}$, and consist of 19 channels of $0.5 \mathrm{~mm}$ radius each $(19 \%$ porosity). We select silicon carbide $(\epsilon=9.72-2.3 j$ at $300 \mathrm{~K})$ and iron oxide $(\epsilon=9.8-1.1 j$ at $300 \mathrm{~K})$, as both are excellent microwave absorbers, to probe their interaction with the microwave cavity. $\mathrm{SiC}$ possesses a high thermal and electrical conductivity $(\kappa \sim 50 \mathrm{~W} / \mathrm{m} \mathrm{K}, \sigma \sim 100 \mathrm{~S} / \mathrm{m})$, whereas iron oxide is a poor thermal conductor and a weak electrical conductor $(\kappa \sim 5 \mathrm{~W} / \mathrm{m} \mathrm{K}, \sigma \sim 5 \mathrm{~S} / \mathrm{m}) .{ }^{44,45}$ Furthermore, we include a material identical to $\mathrm{SiC}$ but with a low electrical conductivity of $\sigma=5 \mathrm{~S} / \mathrm{m}$ to delineate the impact of electrical properties.

The results for silicon carbide, iron oxide, and reduced electrical conductivity silicon carbide are shown in Figure $9 a-$ c. Silicon carbide develops minimal temperature difference $(<3$ $\mathrm{K})$, whereas iron oxide exhibits some temperature difference $(>40 \mathrm{~K})$ due to its low thermal conductivity. We find that electrical conductivity influences heat distribution, as evidenced by the similarity between the temperature fields [Figure 9b,c]. These two structures exhibit radially increasing temperatures toward the center. In contrast, $\mathrm{SiC}$ [Figure 9a] hinders the penetration of microwave radiation, owing to its higher electrical conductivity, and is therefore influenced more strongly by natural convection on the outer surface of the bed, as we conduct these simulations in the absence of flow. Furthermore, the electrical conductivity modifies the electromagnetic radiation in the microwave cavity, changing the temperature. However, the effect is small.

Next, to understand the role of thermal conductivity in temperature inhomogeneity, we plot the minimum, maximum, and average temperatures of structured reactors vs the channel radius in Figure $9 \mathrm{~d}-\mathrm{f}$. The channel radii vary from $0.15 \mathrm{~mm}$ ( $2 \%$ porosity) to $0.75 \mathrm{~mm}$ ( $43 \%$ porosity), at which point the channels begin to touch each other. Despite being heated to nearly identical average temperatures, the iron oxide leads to hot-spots due to its inability to equilibrate as effectively as the higher thermal conductivity counterparts. We note that localized high temperatures are absent in all cases highlighting that structured systems are better suited for microwave 
reaction engineering. A high thermal diffusion is central to having isothermal catalyst structures. With increasing porosity by varying the channel radius, the structured bed effective thermal conductivity diminishes, ${ }^{46}$ evidenced via more considerable temperature inhomogeneity. We note that limited insights are drawn from comparing the raw temperature data upon changing the structure and/or the material as variations in the interactions with the cylindrical microwave cavity (which modifies the coupling frequency for each structurematerial combination) occur.

Thus, structures with high thermal conductivity and without sharp contact points can enable the practical utilization of microwaves for reaction engineering by ensuring a more homogeneous temperature field. A higher electrical conductivity reduces the penetration of microwaves into the structure and provides additional control. Finally, additive manufacturing advances enable structures, such as open foams, ${ }^{47,48}$ with enhanced mass transport, to further accelerate microwave-assisted catalytic applications.

\section{SUMMARY}

We studied the microwave heating of structured beds in a monomodal cylindrical cavity through CFD, experiments, and an analytical model to understand the microwave-induced performance enhancements reported for endothermic reactions. CFD simulations are in good agreement with experiments and provide a nonintrusive tool to assess the entire temperature field, something nearly unattainable experimentally. Consistent with prior work, we demonstrated selective heating, whereby a good microwave absorbing solid material heats preferentially over a fluid. We also showed localized structure heating in the electromagnetic field focus area with minimal upstream fluid heating and low to modest fluid heating downstream caused primarily by convection.

We showed that, for gases with residence times longer than $\sim 0.1 \mathrm{~s}$, it is unlikely to develop a substantial temperature gap throughout the monoliths' length with $\sim 1 \mathrm{~mm}$ sized channels. On the other hand, temperature gradients exist for liquids over longer residence times due to a slow transverse heat transfer. An analytical model and CFD simulations clearly show that, as expected from textbook transport phenomena, the channel dimension and the thermal diffusivity control the transverse heat transfer rate and the thermal equilibration time. In contrast, the flow rate and the structure length dictate the heat removed, the power needed, and the residence time. It is the ratio of two characteristic time scales, the residence time, and the transverse heat transfer, that determines the temperature gradient. A simple order of magnitude analysis provides insights into whether selective heating is responsible for selective catalysis and suppressing gas-phase chemistry. We advocate that localized heating may be more critical than selective heating for selectivity improvement, but future work will be needed to delineate this fully.

Materials of good microwave absorbing properties and high thermal conductivity, e.g., $\mathrm{SiC}$, are well suited for creating 3Dprinted structured reactors with minimal temperature inhomogeneity in the structure. We also reveal that a lower electrical conductivity material allows for a more considerable penetration of microwaves into the structured reactor and better heating. 3D-printed silicon carbide structured reactors can eliminate arcing and create nearly isothermal systems for sustainable electrified chemical manufacturing.

\section{ASSOCIATED CONTENT}

\section{(s) Supporting Information}

The Supporting Information is available free of charge at https://pubs.acs.org/doi/10.1021/acs.iecr.0c05580.

Experimental setup, temperature calibration and control strategy, electrical and thermal properties of $\mathrm{SiC}$, and electric field distribution in the microwave cavity (PDF)

\section{AUTHOR INFORMATION}

\section{Corresponding Author}

Dionisios G. Vlachos - Delaware Energy Institute, University of Delaware, Newark, Delaware 19716, United States; Department of Chemical and Biomolecular Engineering, University of Delaware, Newark, Delaware 19716, United States; ㅇo이.org/0000-0002-6795-8403;

Email: vlachos@udel.edu

\section{Authors}

Abhinav Malhotra - Delaware Energy Institute, University of Delaware, Newark, Delaware 19716, United States

Weiqi Chen - Delaware Energy Institute, University of Delaware, Newark, Delaware 19716, United States

Himanshu Goyal - Department of Chemical Engineering, Indian Institute of Technology Madras, Chennai, Tamil Nadu 600036, India

Pedro J. Plaza-Gonzalez - ITACA Institute, Universitat Politècnica de València, 46022 Valencia, Spain

Ignacio Julian - Instituto de Nanociencia y Materiales de Aragón (INMA), Consejo Superior de Investigaciones Científicas, CSIC-Universidad de Zaragoza, Zaragoza 50009, Spain

Jose M. Catala-Civera - ITACA Institute, Universitat Politècnica de València, 46022 Valencia, Spain

Complete contact information is available at:

https://pubs.acs.org/10.1021/acs.iecr.0c05580

\section{Author Contributions}

A.M. and W.C. contributed equally

Notes

The authors declare no competing financial interest.

\section{ACKNOWLEDGMENTS}

This work was supported by the Department of Energy's Office of Energy Efficiency and Renewable Energy Advanced Manufacturing Office under award no. DE-EE0007888-8.3. The Delaware Energy Institute gratefully acknowledges the State of Delaware's support and partnership toward the RAPID projects. The authors acknowledge fruitful discussions with Prof. Mark Mirotznik, Paul Parsons, and Tai-Ying Chen. The authors thank Keiji Adachi from Ibiden USA for providing the $\mathrm{SiC}$ monolith sample and Jaynell Keely for assistance with graphics.

\section{REFERENCES}

(1) IPCC Report: Global Warming of $1.5^{\circ} \mathrm{C}$; The Intergovernmental Panel on Climate Change, 2018.

(2) Dudukovic, M. P. Frontiers in Reactor Engineering. Science 2009, 325 (5941), 698.

(3) Stankiewicz, A.; Nigar, H. Beyond Electrolysis: Old Challenges and New Concepts of Electricity-Driven Chemical Reactors. React. Chem. Eng. 2020, 5, 1005-1016. 
(4) Van Gerven, T.; Stankiewicz, A. Structure, Energy, Synergy, Time: The Fundamentals of Process Intensification. Ind. Eng. Chem. Res. 2009, 48 (5), 2465-2474.

(5) Dudukovic, M. P. Challenges and Innovations in Reaction Engineering. Chem. Eng. Commun. 2008, 196 (1-2), 252-266.

(6) Van Geem, K. M.; Galvita, V. V.; Marin, G. B. Making Chemicals with Electricity. Science 2019, 364 (6442), 734-735.

(7) Wismann, S. T.; Engbæk, J. S.; Vendelbo, S. B.; Bendixen, F. B.; Eriksen, W. L.; Aasberg-Petersen, K.; Frandsen, C.; Chorkendorff, I.; Mortensen, P. M. Electrified Methane Reforming: A Compact Approach to Greener Industrial Hydrogen Production. Science 2019, 364 (6442), 756-759.

(8) Snoeckx, R.; Bogaerts, A. Plasma Technology - a Novel Solution for CO 2 Conversion? Chem. Soc. Rev. 2017, 46 (19), 5805-5863.

(9) Khattak, H. K.; Bianucci, P.; Slepkov, A. D. Linking Plasma Formation in Grapes to Microwave Resonances of Aqueous Dimers. Proc. Natl. Acad. Sci. U. S. A. 2019, 116 (10), 4000-4005.

(10) Ramirez, A.; Hueso, J. L.; Abian, M.; Alzueta, M. U.; Mallada, R.; Santamaria, J. Escaping Undesired Gas-Phase Chemistry: Microwave-Driven Selectivity Enhancement in Heterogeneous Catalytic Reactors. Sci. Adv. 2019, 5 (3), eaau9000.

(11) Wang, W.; Tuci, G.; Duong-Viet, C.; Liu, Y.; Rossin, A.; Luconi, L.; Nhut, J.-M.; Nguyen-Dinh, L.; Pham-Huu, C.; Giambastiani, G. Induction Heating: An Enabling Technology for the Heat Management in Catalytic Processes. ACS Catal. 2019, 9 (9), 7921-7935.

(12) De Luna, P.; Hahn, C.; Higgins, D.; Jaffer, S. A.; Jaramillo, T. F.; Sargent, E. H. What Would It Take for Renewably Powered Electrosynthesis to Displace Petrochemical Processes? Science 2019, 364 (6438), eaav3506.

(13) Liu, C.; Yu, D.; Kirk, D. W.; Xu, Y. Electromagnetic Wave Absorption of Silicon Carbide Based Materials. RSC Adv. 2017, 7 (2), 595-605.

(14) Stefanidis, G. D.; Muñoz, A. N.; Sturm, G. S. J.; Stankiewicz, A. A Helicopter View of Microwave Application to Chemical Processes: Reactions, Separations, and Equipment Concepts. Rev. Chem. Eng. 2014, 30 (3), 1.

(15) Sturm, G. S. J.; Stankiewicz, A. I.; Stefanidis, G. D. Microwave Reactor Concepts: From Resonant Cavities to Traveling Fields. In Alternative Energy Sources for Green Chemistry; Royal Society of Chemistry, 2016.

(16) Leonelli, C.; Mason, T. J. Microwave and Ultrasonic Processing: Now a Realistic Option for Industry. Chem. Eng. Process. 2010, 49 (9), 885-900.

(17) Sun, J.; Wang, W.; Yue, Q. Review on Microwave-Matter Interaction Fundamentals and Efficient Microwave-Associated Heating Strategies. Materials 2016, 9 (4), 231.

(18) Baker-Jarvis, J.; Kim, S. The Interaction of Radio-Frequency Fields with Dielectric Materials at Macroscopic to Mesoscopic Scales. J. Res. Natl. Inst. Stand. Technol. 2012, 117, 1.

(19) Groppi, G.; Tronconi, E. Honeycomb Supports with High Thermal Conductivity for Gas/Solid Chemical Processes. Catal. Today 2005, 105 (3-4), 297-304.

(20) Tronconi, E.; Groppi, G.; Boger, T.; Heibel, A. Monolithic Catalysts with 'High Conductivity' Honeycomb Supports for Gas/ Solid Exothermic Reactions: Characterization of the Heat-Transfer Properties. Chem. Eng. Sci. 2004, 59 (22-23), 4941-4949.

(21) Razza, S.; Heidig, T.; Bianchi, E.; Groppi, G.; Schwieger, W.; Tronconi, E.; Freund, H. Heat Transfer Performance of Structured Catalytic Reactors Packed with Metal Foam Supports: Influence of Wall Coupling. Catal. Today 2016, 273, 187-195.

(22) Ramírez, A.; Hueso, J. L.; Mallada, R.; Santamaría, J. Ethylene Epoxidation in Microwave Heated Structured Reactors. Catal. Today 2016, 273, 99-105.

(23) Ricciardi, L.; Verboom, W.; Lange, J.; Huskens, J. Reactive Extraction Enhanced by Synergic Microwave Heating: Furfural Yield Boost in Biphasic Systems. ChemSusChem 2020, 13 (14), 3589-3593.

(24) Haneishi, N.; Tsubaki, S.; Abe, E.; Maitani, M. M.; Suzuki, E.; Fujii, S.; Fukushima, J.; Takizawa, H.; Wada, Y. Enhancement of
Fixed-Bed Flow Reactions under Microwave Irradiation by Local Heating at the Vicinal Contact Points of Catalyst Particles. Sci. Rep. 2019, 9 (1), 222.

(25) Horikoshi, S.; Osawa, A.; Sakamoto, S.; Serpone, N. Control of Microwave-Generated Hot Spots. Part V. Mechanisms of Hot-Spot Generation and Aggregation of Catalyst in a Microwave-Assisted Reaction in Toluene Catalyzed by Pd-Loaded AC Particulates. Appl. Catal., A 2013, 460-461, 52-60.

(26) Julian, I.; Pedersen, C. M.; Achkasov, K.; Hueso, J. L.; Hellstern, H. L.; Silva, H.; Mallada, R.; Davis, Z. J.; Santamaria, J. Overcoming Stability Problems in Microwave-Assisted Heterogeneous Catalytic Processes Affected by Catalyst Coking. Catalysts 2019, 9 (10), 867.

(27) Priecel, P.; Lopez-Sanchez, J. A. Advantages and Limitations of Microwave Reactors: From Chemical Synthesis to the Catalytic Valorization of Biobased Chemicals. ACS Sustainable Chem. Eng. 2019, 7 (1), 3-21.

(28) Kappe, C. O. How to Measure Reaction Temperature in Microwave-Heated Transformations. Chem. Soc. Rev. 2013, 42 (12), 4977.

(29) Gangurde, L. S.; Sturm, G. S. J.; Devadiga, T. J.; Stankiewicz, A. I.; Stefanidis, G. D. Complexity and Challenges in Noncontact High Temperature Measurements in Microwave-Assisted Catalytic Reactors. Ind. Eng. Chem. Res. 2017, 56 (45), 13379-13391.

(30) Julian, I.; Ramirez, H.; Hueso, J. L.; Mallada, R.; Santamaria, J. Non-Oxidative Methane Conversion in Microwave-Assisted Structured Reactors. Chem. Eng. J. 2019, 377, 119764.

(31) Nüchter, M.; Ondruschka, B.; Bonrath, W.; Gum, A. Microwave Assisted Synthesis - a Critical Technology Overview. Green Chem. 2004, 6 (3), 128-141.

(32) Muley, P. D.; Nandakumar, K.; Boldor, D. Numerical Modelling of Microwave Heating of a Porous Catalyst Bed. J. Microw. Power Electromagn. Energy 2019, 53 (1), 24-47.

(33) Chen, T.-Y.; Baker-Fales, M.; Vlachos, D. G. Operation and Optimization of Microwave-Heated Continuous-Flow Microfluidics. Ind. Eng. Chem. Res. 2020, 59 (22), 10418.

(34) Goyal, H.; Vlachos, D. G. Multiscale Modeling of MicrowaveHeated Multiphase Systems. Chem. Eng. J. 2020, 397, 125262.

(35) Catala-Civera, J. M.; Canos, A. J.; Plaza-Gonzalez, P.; Gutierrez, J. D.; Garcia-Banos, B.; Penaranda-Foix, F. L. Dynamic Measurement of Dielectric Properties of Materials at High Temperature During Microwave Heating in a Dual Mode Cylindrical Cavity. IEEE Trans. Microwave Theory Tech. 2015, 63 (9), 2905-2914.

(36) Reinosa, J. J.; García-Baños, B.; Catalá-Civera, J. M.; Fernández, J. F. A Step Ahead on Efficient Microwave Heating for Kaolinite. Appl. Clay Sci. 2019, 168, 237-243.

(37) COMSOL Multiphysics, version 5.4; COMSOL AB: Stockholm, Sweden. www.comsol.com.

(38) Horikoshi, S.; Serpone, N. Role of Microwaves in Heterogeneous Catalytic Systems. Catal. Sci. Technol. 2014, 4 (5), 1197.

(39) Bejan, A. Convection Heat Transfer; John Wiley \& Sons, Inc.: Hoboken, NJ, 2013.

(40) Balat-Pichelin, M.; Bousquet, A. Total Hemispherical Emissivity of Sintered $\mathrm{SiC}$ up to $1850 \mathrm{~K}$ in High Vacuum and in Air at Different Pressures. J. Eur. Ceram. Soc. 2018, 38 (10), 34473456.

(41) Slack, G. A. Thermal Conductivity of Pure and Impure Silicon, Silicon Carbide, and Diamond. J. Appl. Phys. 1964, 35 (12), 34603466.

(42) Bird, R. B.; Stewart, W. E.; Lightfoot, E. N. Transport Phenomena; John Wiley \& Sons, 1960; Vol. 413.

(43) Muley, P. D.; Boldor, D. Investigation of Microwave Dielectric Properties of Biodiesel Components. Bioresour. Technol. 2013, 127, $165-174$.

(44) Molgaard, J.; Smeltzer, W. W. Thermal Conductivity of Magnetite and Hematite. J. Appl. Phys. 1971, 42 (9), 3644-3647.

(45) Bidwell, C. C. Electrical and Thermal Properties of Iron Oxide. Phys. Rev. 1917, 10 (6), 756-766. 
(46) Visconti, C. G.; Groppi, G.; Tronconi, E. Accurate Prediction of the Effective Radial Conductivity of Highly Conductive Honeycomb Monoliths with Square Channels. Chem. Eng. J. 2013, 223, 224-230.

(47) Bracconi, M.; Ambrosetti, M.; Okafor, O.; Sans, V.; Zhang, X.; Ou, X.; Da Fonte, C. P.; Fan, X.; Maestri, M.; Groppi, G.; Tronconi, E. Investigation of Pressure Drop in 3D Replicated Open-Cell Foams: Coupling CFD with Experimental Data on Additively Manufactured Foams. Chem. Eng. J. 2019, 377, 120123.

(48) Della Torre, A.; Lucci, F.; Montenegro, G.; Onorati, A.; Dimopoulos Eggenschwiler, P.; Tronconi, E.; Groppi, G. CFD Modeling of Catalytic Reactions in Open-Cell Foam Substrates. Comput. Chem. Eng. 2016, 92, 55-63. 